\title{
On the distribution of energy versus Alfvénic correlation for polar wind fluctuations
}

\author{
B. Bavassano and R. Bruno \\ Istituto di Fisica dello Spazio Interplanetario, Istituto Nazionale di Astrofisica, Rome, Italy \\ Received: 21 July 2006 - Accepted: 18 October 2006 - Published: 22 November 2006
}

\begin{abstract}
Previous analyses have shown that polar wind fluctuations at MHD scales appear as a mixture of Alfvénic fluctuations and variations with an energy imbalance in favour of the magnetic term. In the present study, by separately examining the behaviour of kinetic and magnetic energies versus the Alfvénic correlation level, we unambiguously confirm that the second population is essentially related to a large increase of the magnetic energy with respect to that of the Alfvénic population. The relevant new result is that this magnetic population, though of secondary importance in terms of occurrence frequency, corresponds to a primary peak in the distribution of total energy. The fact that this holds in the case of polar wind, which is the least structured type of interplanetary plasma flow and with the slowest evolving Alfvénic turbulence, strongly suggests the general conclusion that magnetic structures cannot be neglected when modeling fluctuations for all kinds of wind regime.
\end{abstract}

Keywords. Interplanetary physics (MHD waves and turbulence; Sources of the solar wind) - Space plasma physics (Turbulence)

\section{Introduction}

Since the first continuous observations of solar wind performed by Mariner 2 in 1962 it was recognized the existence of a correlation of Alfvénic type between velocity and magnetic field fluctuations (Coleman, 1967; Unti and Neugebauer, 1968). At almost the same time, analyses of 19651966 Pioneer 6 data gave evidence of the filamentary structure of the interplanetary magnetic field (Bartley et al., 1966; McCracken and Ness, 1966), described as bundles of magnetic tubes of force embedded in the solar wind. Thus, already at the very beginning of the space exploration the main ingredients of the solar wind variability at MHD scales were well identified.

Correspondence to: B. Bavassano

(bavassano@ifsi-roma.inaf.it)
In the next decades impressive advances have been done thanks to the increasing quality of plasma and magnetic field measurements, performed on extended ranges of solar distance and latitude in the heliosphere. Fundamental contributions have been also given by new analysis methods and use of advanced concepts to describe the behaviour of solar wind fluctuations. Several reviews offer exhaustive discussions about all this (e.g., Goldstein et al., 1995a; Tu and Marsch, 1995; Bavassano et al., 2004; Bruno and Carbone, 2005).

Still, there are points that need further analyses. One is that of the energy distribution among the various components of solar wind fluctuations. In particular, the question addressed here is that of how the kinetic and magnetic energies are distributed between Alfvénic and non-Alfvénic disturbances or, versus the level of the Alfvénic correlation.

Past studies (e.g., Bavassano et al., 1998) have shown how the normalized residual energy (defined as the difference between kinetic and magnetic energies divided by their sum, see section on data analysis) varies with the Alfvénic correlation. In the present study, instead of using a normalized quantity, we will directly look at the kinetic and magnetic energy values. This will be done for two different samples of the polar solar wind. This is the wind that is typically observed in the high-latitude heliosphere for conditions of low solar activity. A fast, teneous, and steady flow, as compared to low-latitude conditions, the polar wind is the best example in the heliosphere of an almost structureless flow. As typical of the fast wind regime (Tu and Marsch, 1995), the polar wind is characterized by the presence of a strong flow of fluctuations of Alfvénic type (e.g., Goldstein et al., 1995b; Smith et al., 1995; Bavassano et al., 2000).

\section{Data analysis}

Our analysis is based on data of the solar wind plasma and magnetic field experiments aboard Ulysses, the first spacecraft that, with a highly inclined orbital plane with respect to the ecliptic, has been able to explore the heliosphere up

Published by Copernicus GmbH on behalf of the European Geosciences Union. 
Table 1. Start and end times (year, day, hour), minimum and maximum distances $(R$, in $\mathrm{AU})$, and latitudes $(\lambda$, in degrees, northern hemisphere) of the core (first row) and boundary (second row) interval.

\begin{tabular}{ccc}
\hline time interval & ${ }^{R}$ & ${ }^{\lambda}{ }^{\lambda} \max$ \\
& $\min ^{\max }$ \\
\hline $199517613-199526017$ & $1.77-2.36$ & $72.5-80.2$ \\
$199610617-199618223$ & $3.65-4.02$ & $32.8-40.1$ \\
\hline
\end{tabular}

to $80^{\circ}$ of latitude, with extended full immersions in the polar wind. The available plasma data are the fluid velocity vector (averaged over proton and alpha-particle populations), the proton number density, the alpha-particle number density, and the proton temperature. The time resolution is either 4 or 8 min, depending on the spacecraft mode of operation. As regards the magnetic field, the data used are 1-min averages of both components and magnitude, computed from higher resolution measurements.

In the present study we will use two samples selected from the northern leg of the Ulysses' first out-of-ecliptic orbit, when the polar wind is exceptionally stable (McComas al., 2000). Both intervals are three solar rotations long, as seen by Ulysses. Their time, distance, and latitude ranges are given in Table 1. The interval of the first row, in the following indicated as "core" interval, corresponds to a top-latitude cut of the Ulysses' trajectory, while that of the second row, in the following indicated as "boundary" interval, is from the distant polar wind, near the low-latitude boundary of the polar flow.

Before describing the analysis method we would like to recall that two different Alfvénic populations are usually present in the solar wind (e.g., see review by Tu and Marsch, 1995), one of fluctuations propagating, in the wind plasma frame, away from Sun (outward population) and the other of fluctuations propagating towards the Sun (inward population). Outside the Alfvénic critical point, where the solar wind becomes super-Alfvénic, both kinds of fluctuation are obviously convected outwards as seen from the Sun. Generally most of the energy comes from the outward population. The Sun is the major source for outward fluctuations, with smaller contributions from interplanetary sources. Conversely, inward fluctuations can only come from sources outside the Alfvénic critical point, since inside this point inward waves fall back to the Sun (for the completeness it should be mentioned that they might originate inside the critical point as well, if they initially propagate outward and then are scattered backward due to some non-linear process).

The computational scheme of our analysis is simple. In a first step, for each plasma velocity vector $\boldsymbol{V}$ we have determined the corresponding magnetic field vector $\boldsymbol{B}$ by averaging over 4 (or 8) min, then have derived the corre-
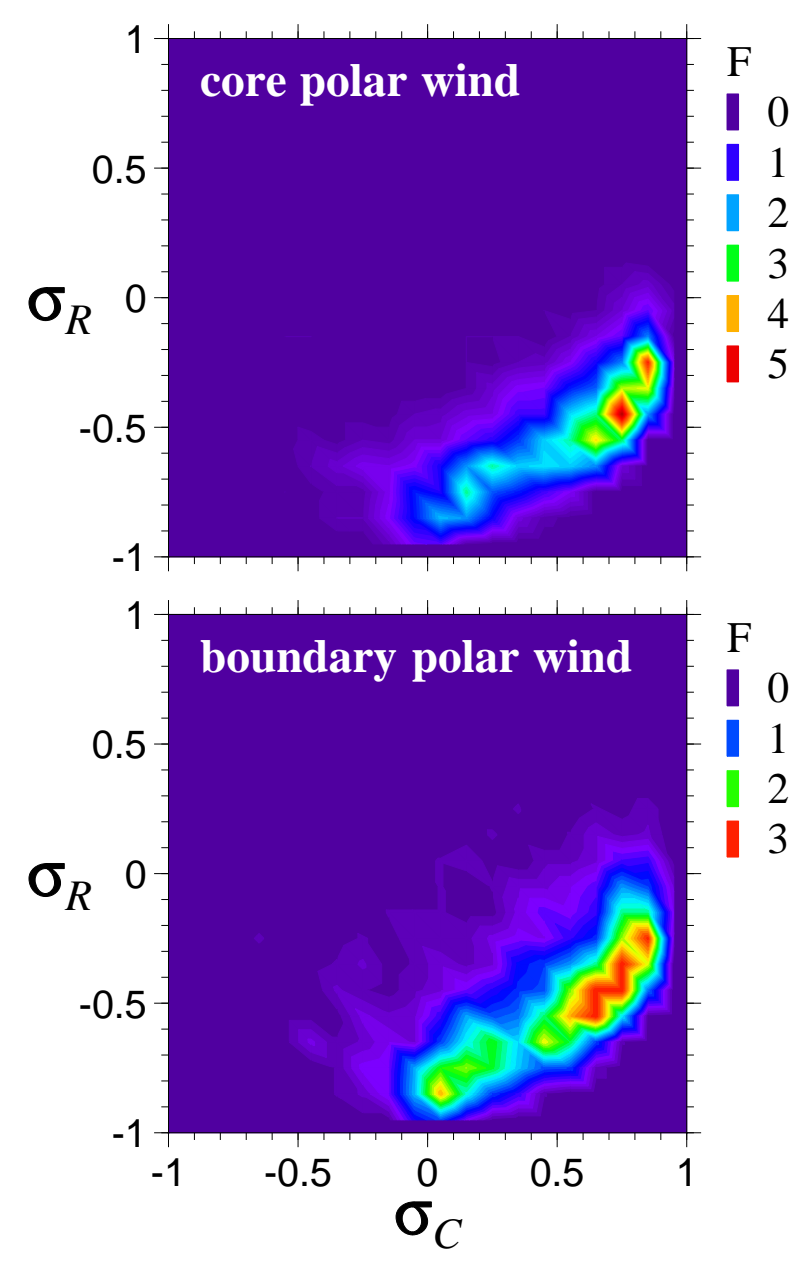

Fig. 1. Distribution of the occurrence frequency $F$ (in per cent) in the plane $\sigma_{C}-\sigma_{R}$ for core (top) and boundary (bottom) polar wind intervals. Note that the scale for $F$ is different in the two panels.

sponding Elsässer's variables $\boldsymbol{Z}_{ \pm}$(Elsässer, 1950), defined as $\boldsymbol{V} \pm \boldsymbol{B} / \sqrt{4 \pi \rho}$ (with $\rho$ the mass density, computed by including both proton and alpha-particle contributions). These variables are ideally suited to separately identify Alfvénic fluctuations propagating in opposite directions (e.g., see Tu and Marsch (1995) for a discussion on their use). When studying Alfvénic fluctuations in solar wind, it is useful to have that $\boldsymbol{Z}_{+}\left(\boldsymbol{Z}_{-}\right)$fluctuations always correspond to an outward (inward) propagation, whatever the direction of the background magnetic field is. This request is fulfilled by applying the above definition only when the background field has a sunward component along the local spiral direction, while for the opposite magnetic polarity $\boldsymbol{Z}_{ \pm}$is taken as $\boldsymbol{V} \mp \boldsymbol{B} / \sqrt{4 \pi \rho}$. In the following we will apply this dual definition. Obviously, in the case of folded (or S-shaped) field configurations, erroneous outward/inward classifications are obtained. This, however, occurs for a quite small number of cases (see Balogh et al., 1999). 


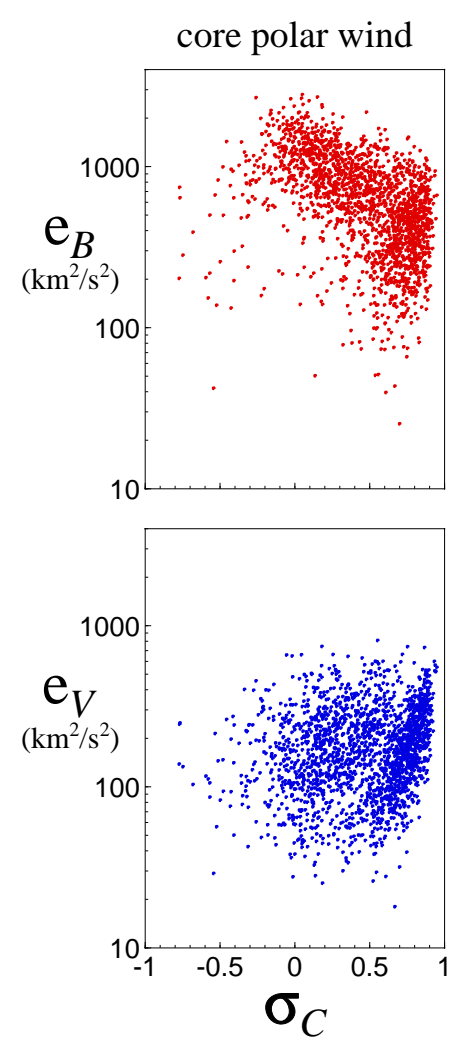

boundary polar wind
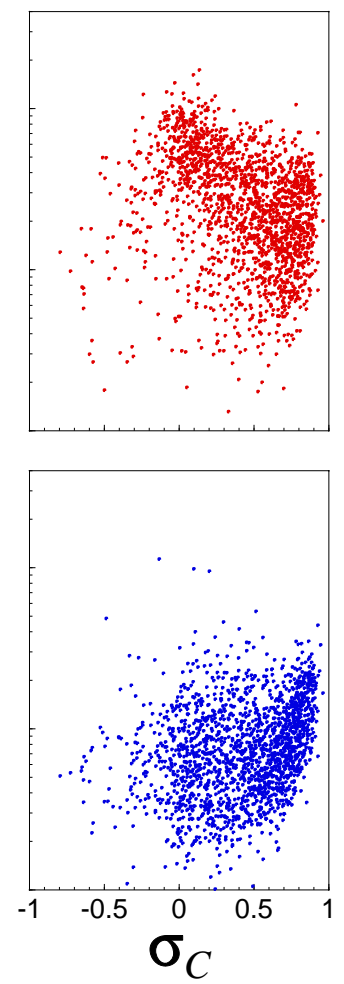

Fig. 2. $\sigma_{C}-e_{B}$ (top) and $\sigma_{C}-e_{V}$ (bottom) scatter plots for core (left) and boundary (right) polar wind intervals.

In the next step, from the time series of $\boldsymbol{V}, \boldsymbol{B} / \sqrt{4 \pi \rho}, \boldsymbol{Z}_{+}$, and $\boldsymbol{Z}_{-}$we have computed the corresponding total variances $e_{V}, e_{B}, e_{+}$, and $e_{-}$, as given by the trace of the variance matrices. These values give a measure of the energy (per unit mass) associated to the various fluctuating fields in a given frequency band, determined by the data sampling time (see above) and by the averaging time used to evaluate variances. Results discussed here refer to hourly variances. With this choice we essentially focus on fluctuations in the core of the inertial Alfvénic regime (e.g., Goldstein et al., 1995b; Horbury et al., 1995). We would like to underline that, as already mentioned, the computed energies are for unit mass. In particular, when dealing with the magnetic energy $e_{B}$, one has to keep in mind that it is the variance of $\boldsymbol{B} / \sqrt{4 \pi \rho}$, namely the magnetic field scaled to Alfvén units or, the Alfvén speed.

Finally, from the above quantities we have derived two normalized differences, namely 1) the normalized residual energy $\sigma_{R}=\left(e_{V}-e_{B}\right) /\left(e_{V}+e_{B}\right)$, a measure of the energy sharing between kinetic and magnetic fluctuations, and 2) the normalized cross-helicity $\sigma_{C}=\left(e_{+}-e_{-}\right) /\left(e_{+}+e_{-}\right)$, a measure of the energy sharing between the two components (outward and inward) of the Alfvénic fluctuations. The normalized cross-helicity gives also a measure of the level of correlation between velocity and magnetic fluctuations (Roberts

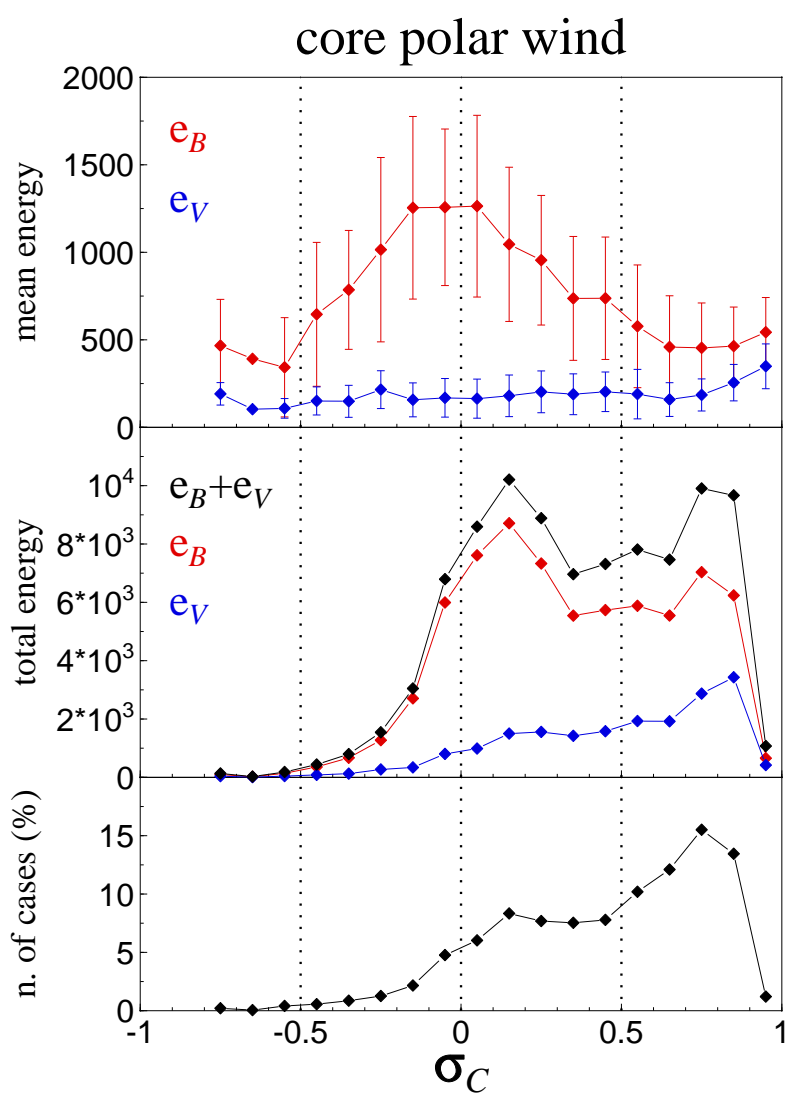

Fig. 3. Energy distributions versus cross-helicity for core polar wind. The top and middle panels give mean and total values of the magnetic (red) and kinetic (blue) energies (in $\mathrm{km}^{2} / \mathrm{s}^{2}$ ) for 0.1-bin of $\sigma_{C}$, respectively. In the top panel error bars are standard deviations. In the middle panel the black line refers to the sum of magnetic and kinetic energies. The bottom panel gives the number of cases (in per cent) for each $\sigma_{C}$ bin.

et al., 1987). In fact (Bavassano et al., 1998), provided that $\sigma_{R} \neq \pm 1$, the correlation coefficient is $\sigma_{C} / \sqrt{1-\sigma_{R}^{2}}$ (note the constraint $\sigma_{C}^{2}+\sigma_{R}^{2} \leq 1$ ).

\section{Magnetic and kinetic energies versus cross-helicity}

Figure 1 shows 2-D frequency distributions of $\sigma_{C}$ and $\sigma_{R}$ hourly values for the analysed polar wind intervals. As expected on the basis of previous analyses by Bavassano et al. $(1998,2005)$, in both cases essentially the same pattern is observed, with a prominent peak near $\sigma_{C} \sim 0.8$ and $\sigma_{R} \sim-0.5$ and a tail extending towards $\sigma_{C} \sim 0$ and $\sigma_{R} \sim-1$, with a secondary peak near the end. The main peak is easily interpreted in terms of wind regions dominated by outward $\left(\boldsymbol{Z}_{+}\right)$Alfvénic fluctuations, while the secondary peak corresponds to an ensemble of fluctuations with a strong energy imbalance in favour of the magnetic term. Not surprisingly (e.g., see Bavassano et al., 2000), for the core sample the 


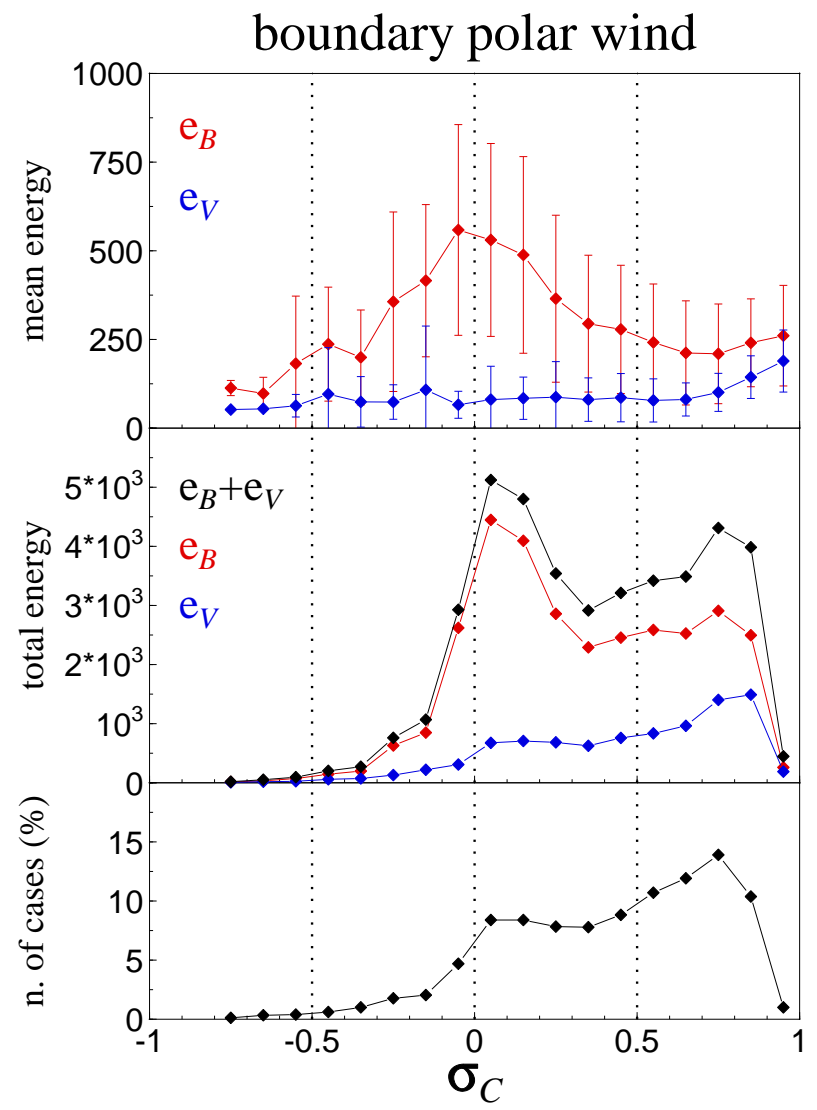

Fig. 4. The same as in Fig. 3, for boundary polar wind.

Alfvénic peak is more pronounced than for the boundary sample.

Obviously, plots as those in Fig. 1 do not allow to understand if cases falling near $(0,-1)$ in the $\sigma_{C}-\sigma_{R}$ plane have to be related to an increase of magnetic fluctuations, or to a decline of velocity fluctuations, or to both events. Moreover, from a normalized quantity as $\sigma_{R}$ it is impossible to understand the relative weight, in terms of energy content, of the different kinds of fluctuation. These points are addressed in Fig. 2, where scatter plots of cross-helicity and energy values are given in $\sigma_{C}-e_{B}$ (top) and $\sigma_{C}-e_{V}$ (bottom) planes for the core (left) and boundary (right) polar sample. The energy levels are different in the two samples (higher in core polar wind), but the overall pattern is the same, with the decrease of cross-helicity (in absolute value) essentially associated to a robust increase of the magnetic energy $e_{B}$. In the following we will indicate this ensemble of fluctuations as "magnetic population".

To further investigate these features the -1 to $1 \sigma_{C}$ range has been divided into 20 bins and for each subset of data falling in a bin we have computed means, standard deviations, and total values of magnetic and kinetic energy. Total values are defined as product of the mean value by the per-

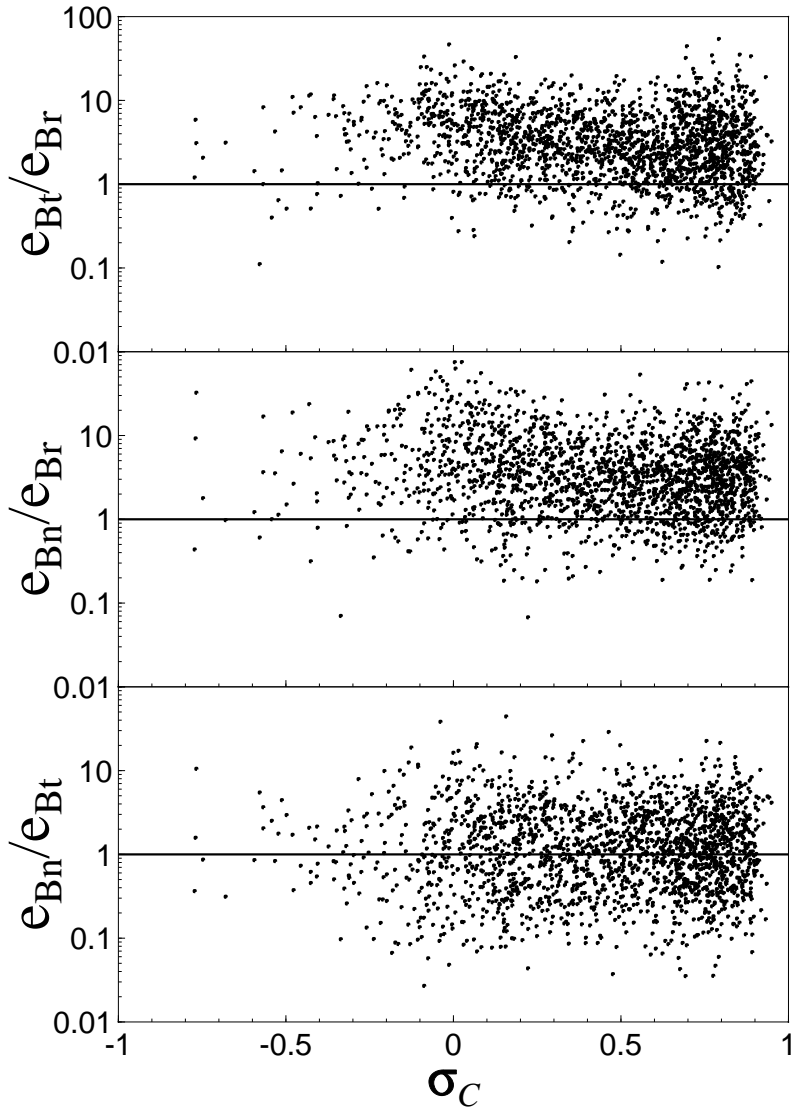

Fig. 5. Geometrical properties of the magnetic fluctuations versus cross-helicity for the core polar wind sample. Plotted data are the ratios between values of $e_{B}$ along the $r t n$ frame axes (see definition in the text).

centage of cases in the bin (this makes meaningful a comparison between total energies from samples with a different number of data). Plots of all these quantities are given in Figs. 3 and 4 for the core and boundary interval, respectively. The top panel of each figure shows the variation with $\sigma_{C}$ of the mean values, with standard deviations as error bars (red for magnetic energy, blue for kinetic energy). The middle panel is for the total $e_{B}$ and $e_{V}$ values (again red and blue lines, respectively) and for their sum (black line). The bottom panel gives the percentage of cases for each $\sigma_{C}$ bin.

Again, trends are similar in the two figures, though with different energy levels (scales are higher of a factor of two for the core sample). The curves in the top panels confirm that low (in absolute value) cross-helicity corresponds to a strong increase in the energy of fluctuations of magnetic type (velocity fluctuations only have a weak decrease for $\sigma_{C}$ from $\sim 1$ to $\sim 0.7$ ). A second point to be stressed is that (see middle panels) the low cross-helicity population, though a secondary peak in terms of number of cases, is a major peak in the distribution of total magnetic energy (red curve). Given 
the low level of total kinetic energy, this also holds for the sum of total energies (black curve). Thus, from the point of view of the energy content of solar wind fluctuations, the magnetic population is seen to play a primary role. It is worthy of note that this feature is more pronounced for the nearboundary, and more distant, polar wind.

\section{Geometrical properties of the magnetic fluctuations versus cross-helicity}

Given the predominant role of the magnetic fluctuations it seems useful to further characterize them by looking at a possible dependence on $\sigma_{C}$ of their geometrical properties. This has been done for the core polar wind sample, when the background magnetic field is nearly radial (average spiral angle of $8.5^{\circ}$ ). In a first approximation, this geometry allows to separate parallel and perpendicular (to the background field) fluctuations by directly using the $r t n$ reference axes of the Ulysses data ( $r$ outwards along the Sun-spacecraft line, $n$ northwards in the plane defined by $r$ and solar rotation axis, and $t$ to complete a right-handed system).

Figure 5 shows the ratios between $e_{B r}, e_{B t}$, and $e_{B n}$ (the $e_{B}$ values along $r, t$, and $n$ axes, respectively) as a function of $\sigma_{C}$. For all the $\sigma_{C}$ range the magnetic fluctuations appear characterized by 1) an equipartition between transverse fluctuations (see $e_{B n} / e_{B t}$ in the bottom panel) and 2) a predominance of these fluctuations with respect to the radial ones (see $e_{B t} / e_{B r}$ and $e_{B n} / e_{B r}$ in top and middle panel, respectively). It is worthy of note that this last feature becomes more pronounced as $\sigma_{C}$ goes to 0 . In other words, for low cross-helicity the fluctuations tend to be more planar (in a plane nearly perpendicular to the background magnetic field) than for cross-helicity approaching 1 .

\section{Discussion and conclusion}

Previous studies (Bavassano et al., 1998, 2005) have shown that polar wind fluctuations at hourly scale essentially are a mixture of 1) variations of Alfvénic type and 2) variations characterized by $\sigma_{C} \sim 0$ and $\sigma_{R} \sim-1$.

In the present analysis, by separately examining the behaviour of $e_{B}$ and $e_{V}$ versus $\sigma_{C}$, we have unambiguously confirmed that the second population is essentially related to a large increase of the magnetic energy with respect to the levels typically observed for the Alfvénic population.

The relevant new result is that this magnetic population, though of secondary importance in terms of occurrence frequency, corresponds to a primary peak in the distribution of total (magnetic plus kinetic) energy. Thus, from the point of view of the energy content of solar wind fluctuations, the magnetic population plays a primary role.

As regards geometrical properties, the magnetic population appears to be made of fluctuations more planar, in a plane roughly perpendicular to the background magnetic field, than those of the Alfvénic population. This is reminiscent of quasi-two-dimensional fluctuations, first discussed by Matthaeus et al. (1990) to account for the Maltese-cross shape of the 2-D magnetic correlation function (with the caveat that no analysis is done here for the propagation vectors, that for 2-D fluctuations have to be transverse to both the background and the fluctuating fields). Yet, it should be noted that the observed geometry may well come from coronal magnetic flux tubes that, turbulently mixed, are swept out by the solar wind (e.g., see Thieme et al., 1990). Planar magnetic structures embedded in the solar wind were discussed in detail by Tu and Marsch (1991), who named them "magnetic field directional turnings". A recent study of Bruno et al. (submitted, 2006) ${ }^{1}$ shows how remarkable their role is for solar wind variability.

In conclusion, though the polar wind is a weakly structured plasma flow (e.g., McComas et al., 2000) with a slowly evolving Alfvénic turbulence (Goldstein et al., 1995b; Horbury et al., 1995), in the hourly-scale domain the ensemble of magnetic structures appears to have a total energy content comparable to that of the Alfvénic population. This strongly supports the view that magnetic structures cannot be neglected when modeling MHD fluctuations for all kinds of wind regime. Efforts done in the past (Tu and Marsch, 1993; Schmidt and Marsch, 1995; Schmidt, 1995) to combine the effects of Alfvén waves and convective structures should be further developed.

Acknowledgements. The use of data of the plasma analyzer (principal investigator D. J. McComas, Southwest Research Institute, San Antonio, Texas, USA) and of the magnetometers (principal investigator A. Balogh, The Blackett Laboratory, Imperial College, London, UK) aboard the Ulysses spacecraft is gratefully acknowledged. The data have been made available through the World Data Center A for Rockets and Satellites (NASA/GSFC, Greenbelt, Maryland, USA). We are indebted to G. Consolini for very fruitful discussions. The present work has been supported by the Italian Space Agency (ASI) under contract I/035/05/0 ASI/INAF-OATO.

Topical Editor B. Forsyth thanks G. Erdös and another referee for their help in evaluating this paper.

\section{References}

Balogh, A., Forsyth, R. J., Lucek, E. A., and Horbury, T. S.: Heliospheric magnetic field polarity inversions at high heliographic latitudes, Geophys. Res. Lett., 26, 631-634, 1999.

Bartley, W. C., Bukata, R. P., McCracken, K. G., and Rao, U. R.: Anisotropic cosmic radiation fluxes of solar origin, J. Geophys. Res., 71, 3297-3304, 1966.

Bavassano, B., Bruno, R., and Carbone, V.: MHD turbulence in the heliosphere: evolution and intermittency, in: The Sun and

\footnotetext{
${ }^{1}$ Bruno, R., D’Amicis, R., Bavassano, B., Carbone, V., and Sorriso-Valvo, L.: Magnetic field directional turnings as an important component of the solar wind turbulence, Ann. Geophys., 2006.
} 
the heliosphere as an integrated system, edited by: Poletto, G. and Suess, S. T., Kluwer Academic Publishers, Dordrecht, The Netherlands, Astrophysics and Space Science Library, 317, 253282, 2004.

Bavassano, B., Pietropaolo, E., and Bruno, R.: Cross-helicity and residual energy in solar wind turbulence: radial evolution and latitudinal dependence in the region from 1 to $5 \mathrm{AU}$, J. Geophys. Res., 103, 6521-6529, 1998.

Bavassano, B., Pietropaolo, E., and Bruno, R.: On the evolution of outward and inward Alfvénic fluctuations in the polar wind, J. Geophys. Res., 105, 15 959-15 964, 2000.

Bavassano, B., Pietropaolo, E., and Bruno, R.: Alfvénic fluctuations in "newborn" polar solar wind, Ann. Geophys., 23, 1513-1520, 2005 , http://www.ann-geophys.net/23/1513/2005/.

Bruno, R. and Carbone, V.: The solar wind as a turbulence laboratory, Living Rev. Solar Phys. 2, also available: http://www. livingreviews.org/lrsp-2005-4, 2005.

Coleman Jr., P. J.: Wave-like phenomena in the interplanetary plasma: Mariner 2, Planet. Space Sci., 15, 953-973, 1967.

Elsässer, W. M.: The hydromagnetic equations, Phys. Rev., 79, 183-183, 1950.

Goldstein, M. L., Roberts, D. A., and Matthaeus, W. H.: Magnetohydrodynamic turbulence in the solar wind, Annu. Rev. Astron. Astrophys., 33, 283-325, 1995a.

Goldstein, B. E., Smith, E. J., Balogh, A., Horbury, T. S., Goldstein, M. L., and Roberts, D. A.: Properties of magnetohydrodynamic turbulence in the solar wind as observed by Ulysses at high heliographic latitudes, Geophys. Res. Lett., 22, 3393-3396, 1995 b.

Horbury, T. S., Balogh, A., Forsyth, R. J., and Smith, E. J.: Observations of evolving turbulence in the polar solar wind, Geophys. Res. Lett., 22, 3401-3404, 1995.

Matthaeus, W. H., Goldstein, M. L., and Roberts, D. A.: Evidence for the presence of quasi-two-dimensional nearly incompressible fluctuations in the solar wind, J. Geophys. Res., 95, $20673-$ $20683,1990$.
McComas, D. J., Barraclough, B. L., Funsten, H. O., Gosling, J. T., Santiago-Muñoz, E., Skoug, R. M., Goldstein, B. E., Neugebauer, M., Riley, P., and Balogh, A.: Solar wind observations over Ulysses first full polar orbit, J. Geophys. Res., 105(A5), 10 419-10 433, 2000.

McCracken, K. G. and Ness, N. F.: The collimation of cosmic rays by the interplanetary magnetic field, J. Geophys. Res., 71, 33153318, 1966.

Roberts, D. A., Klein, L. W., Goldstein, M. L., and Matthaeus, W. H.: The nature and evolution of magnetohydrodynamic fluctuations in the solar wind: Voyager observations, J. Geophys. Res., 92, $11021-11040,1987$.

Schmidt, J. M.: Spatial transport and spectral transfer of solar wind turbulence composed of Alfvén waves and convective structures 2: Numerical results, Ann. Geophys., 13, 475-493, 1995, http://www.ann-geophys.net/13/475/1995/.

Schmidt, J. M. and Marsch, E.: Spatial transport and spectral transfer of solar wind turbulence composed of Alfvén waves and convective structures 1: The theoretical model, Ann. Geophys., 13, 459-474, 1995, http://www.ann-geophys.net/13/459/1995/.

Smith, E. J., Balogh, A., Neugebauer, M., and McComas, D. J.: Ulysses observations of Alfvén waves in the southern and northern solar hemispheres, Geophys. Res. Lett., 22, 3381-3384, 1995.

Thieme, K. M., Marsch, E., and Schwenn, R.: Spatial structures in high-speed streams as signatures of fine structures in coronal holes, Ann. Geophys., 8, 713-723, 1990, http://www.ann-geophys.net/8/713/1990/.

Tu, C.-Y. and Marsch, E.: A case study of very low cross-helicity fluctuations in the solar wind, Ann. Geophys., 9, 319-332, 1991, http://www.ann-geophys.net/9/319/1991/.

Tu, C.-Y. and Marsch, E.: A model of solar wind fluctuations with two components: Alfvén waves and convective structures, J. Geophys. Res., 98, 1257-1276, 1993.

Tu, C.-Y. and Marsch, E.: MHD structures, waves and turbulence in the solar wind: Observations and theories, Space Sci. Rev., 73, $1-210,1995$.

Unti, T. W. J. and Neugebauer, M.: Alfvén waves in the solar wind, Phys. Fluids, 11, 563-568, 1968. 